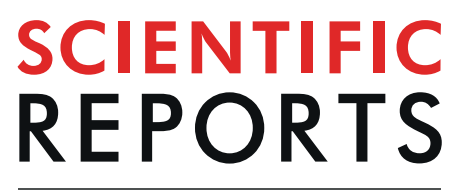

natureresearch

\title{
Early Recognition of the Preference for Exclusive Breastfeeding in Current China: A Prediction Model based on Decision Trees
}

\author{
Yiting Wang ${ }^{1,2,5}$, Chunjian Shan ${ }^{1,2,3,5}$, Yingying Zhang ${ }^{2}$, Lei Ding ${ }^{2}$, Juan Wen ${ }^{4}$ \& \\ Yingying $\operatorname{Tian}^{1,2 *}$
}

Exclusive breastfeeding (EBF) is affected by multiple risk factors. Therefore, it is difficult for clinical professionals to identify women who will not practice EBF well and provide subsequent medical suggestions and treatments. This study aimed to apply a decision tree (DT) model to predict EBF at two months postpartum. The socio-demographic, clinical and breastfeeding parameters of 1,141 breastfeeding women from Nanjing were evaluated. Decision tree modelling was used to analyse and screen EBF factors and establish a risk assessment model of EBF. The Chinese version of the Breastfeeding Self-Efficacy Scale (CV-BSES) score, early formula supplementation, abnormal nipples, mastitis, neonatal jaundice, cracked or sore nipples and intended duration of breastfeeding were significant risk factors associated with EBF in the DT model. The accuracy, sensitivity and specificity of the DT model were $73.1 \%, 75.5 \%$ and $66.3 \%$, respectively. The DT model showed similar or better performance than the logistic regression model in assessing the risk of early cessation of EBF before two months postpartum. The DT model has potential for application in clinical practice and identifies highrisk subpopulations that need specific prevention.

Breast milk is the safest and most nutritious food for infants ${ }^{1}$. Exclusive breastfeeding during the first six months, as well as the continuation of breastfeeding with additional food for two years or longer, is recommended by the World Health Organization (WHO) and United Nations Children's Fund (UNICEF) ${ }^{1,2}$. Breastfeeding not only reduces morbidity and mortality but also has multiple long-term benefits for both children and mothers. For example, breastfeeding can reduce the incidence of obesity and non-communicable diseases and is consistently associated with higher performance in intelligence tests in children and adolescents ${ }^{3-6}$. The maternal benefits of breastfeeding include a more rapid return of postpartum uterine tone, postpartum weight loss, delayed resumption of menses, and decreased risks of breast, ovarian, and endometrial cancers ${ }^{3-6}$. Moreover, breastfeeding also strengthens the infant-mother bond and reduces or eliminates the cost of purchasing formula ${ }^{6}$. Despite the proven benefits of exclusive breastfeeding, many mothers cease the practice prematurely.

Breastfeeding is affected by a complex combination of factors, such as maternal sociodemographic factors, health care, psychosocial factors, type and availability of social support, and public policy. Numerous factors have been shown to influence the duration of exclusive breastfeeding (EBF) and the exclusiveness of breastfeeding, such as parity ${ }^{7}$, maternal education ${ }^{7-9}$, age ${ }^{7,9}$, employment $^{9-11}$, smoking $^{12}$, antenatal classes ${ }^{7,13}$, body mass index ${ }^{14}$, pacifier use ${ }^{7,15}$, and the intended duration of breastfeeding. ${ }^{9}$. Psychological factors have also been linked to the duration of breastfeeding ${ }^{16}$. All of the breastfeeding factors are variable due to culture and perinatal period differences. Therefore, it is difficult for clinical nurses and breastfeeding professionals to identify women who will not practice exclusive breastfeeding well and provide effective interventions.

\footnotetext{
${ }^{1}$ Department of Obstetrics, Women's Hospital of Nanjing Medical University, Nanjing Maternity and Child Health Care Hospital, Nanjing, 210004, China. ${ }^{2}$ Nanjing Maternity and Child Health Care Institute, Women's Hospital of Nanjing Medical University, Nanjing Maternity and Child Health Care Hospital, Nanjing, 210004, China. ${ }^{3}$ Committee of Obstetrics and Gynaecology, Nursing Association of Jiangsu Province, Nanjing, 210004, China. ${ }^{4}$ State Key Laboratory of Reproductive Medicine, Women's Hospital of Nanjing Medical University, Nanjing Maternity and Child Health Care Hospital, Nanjing, 210004, China. ${ }^{5}$ These authors contributed equally: Yiting Wang and Chunjian Shan. *email: tianyingying@njmu.edu.cn
} 


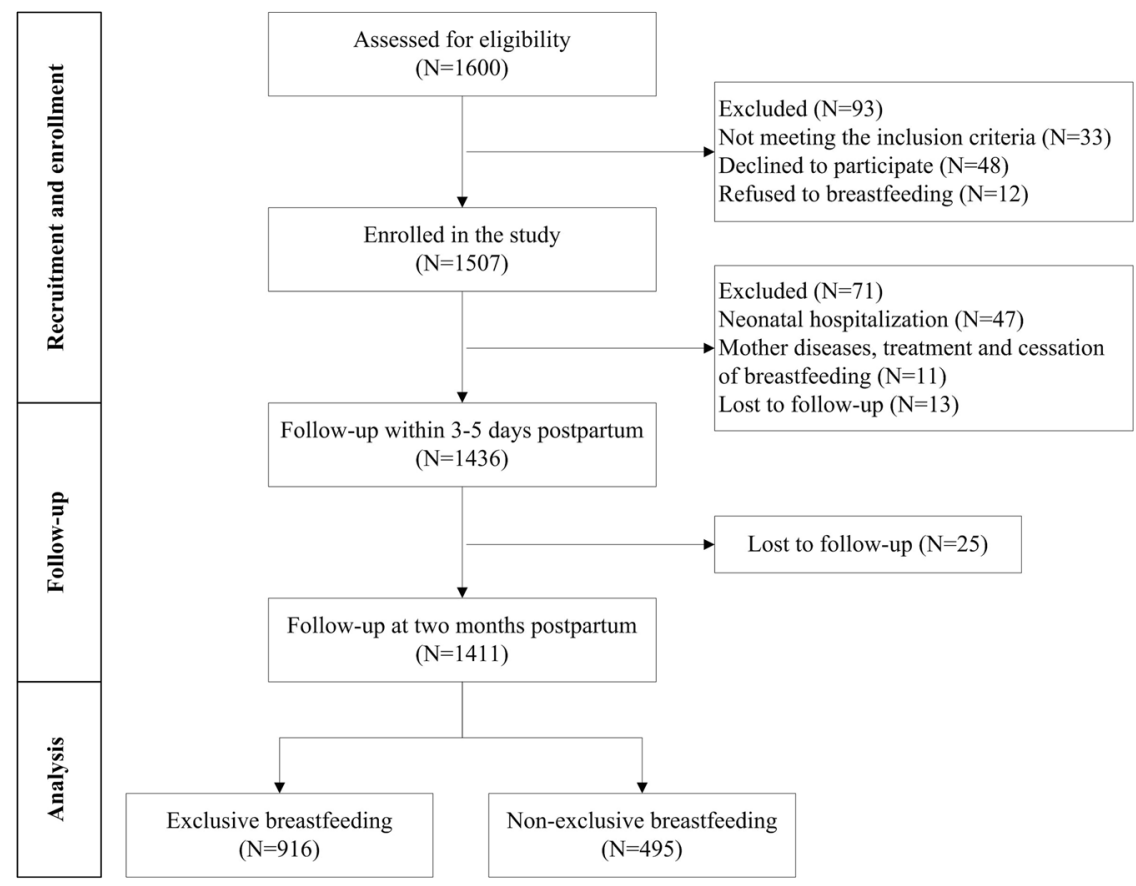

Figure 1. Flow diagram for the inclusion and exclusion criteria.

Breastfeeding in China has other unique features in addition to having a homogeneous population because Chinese society has undergone a rapid surge and transition. In China, 79.6\% of infants were ever breastfed, and $20.8 \%$ of infants were exclusively breastfed at 6 months. However, only $11.5 \%$ and $6.9 \%$ continued to be breastfed at 1 and 2 years, respectively ${ }^{17}$. Jiangsu Province is one of the most economically developed coastal provinces in China. The urban population of Jiangsu Province reached 476.76 million, and its urbanization rate and economic aggregate are among the highest China. Additionally, Nanjing is the capital of Jiangsu Province, where the migrant population accumulates from all over Jiangsu Province. The acceleration of urbanization and economic development may have affected the practice of breastfeeding ${ }^{18}$. For example, due to the high housing price, young parents are forced to live with their parents. The influence of grandmothers on breastfeeding practice is mixed. Meanwhile, breastfeeding mothers must return to work early because of inadequate maternity protection in informal work sectors. The increased marketing of breast milk substitutes may have contributed to the decrease in breastfeeding.

The decision tree (DT) method is a data mining technology that has been widely applied in medicine and public health ${ }^{19-22}$. The DT model is a machine learning model comprising decision rules based on optimal feature cut-off values that recursively split independent variables into different groups to predict an outcome hierarchically. The decision tree can be used to transform a complex decision process based on the influences of different factors into a series of simple decisions ${ }^{23}$. The decision tree divides samples according to the best predictors of the results to provide risk assessments ${ }^{19}$. Furthermore, the DT method is a powerful statistical tool for classifying, predicting, interpreting, and processing data. The algorithm is non-parametric and can efficiently manage large, complex datasets without imposing a complex parametric structure. Missing values and heavily skewed data are easily managed without needing data transformation ${ }^{24}$. The computer-generated model is graphically represented as a tree structure that is easy to interpret and use in clinical settings compared with the OR values of the logistic regression (LR) model ${ }^{25}$.

The aim of this study was to investigate the risk factors involved in the early cessation of EBF and construct a prediction model based on a decision tree (DT) model that can provide a tool and improve clinical professionals' ability to identify women at risk for discontinuing breastfeeding before two months postpartum. Additionally, the performance of the DT model was studied.

\section{Results}

Participant characteristics. Among the 1,507 recruited participants, 1,411 women completed the study (retention rate $=93.6 \%$ ). Meanwhile, 71 participants withdrew at 3-5 days postpartum, and 25 participants withdrew at the end of two months postpartum; the reasons for withdrawal are shown in Fig. 1. Among the 1,411 participants, 495 (35\%) discontinued exclusive breastfeeding before two months postpartum. The rate of exclusive breastfeeding at two months postpartum was $64.9 \%$. The socio-demographic, clinical and breastfeeding characteristics of the participants are presented in Table 1. Overall, the mean age of all the participants was $29.5 \pm 3.49$ (range:18-45) years. Most of the participants preferred to be primiparous (78.7\%), had a vaginal delivery (63.7\%), had a college degree $(73.4 \%)$, worked full-time during pregnancy $(88.7 \%)$, had a medium level of household income per year (75.5\%), did not drink or smoke during the pregnancy (98.9\%), lived with their parents $(75.0 \%)$ and returned to work before 6 months postpartum (84.3\%). 


\begin{tabular}{|c|c|c|c|c|c|}
\hline \multirow[b]{2}{*}{ Variable } & \multicolumn{2}{|c|}{ Exclusive breastfeeding } & \multirow[b]{2}{*}{ OR } & \multirow[b]{2}{*}{$95 \%$ CI } & \multirow[b]{2}{*}{ P-value } \\
\hline & Yes, $N=916,(\%)$ & No, $\mathrm{N}=495,(\%)$ & & & \\
\hline Age, years & $29.48 \pm 3.41$ & $29.51 \pm 3.53$ & 1.002 & $0.971-1.034$ & 0.901 \\
\hline Gestation, weeks & $39.66 \pm 1.08$ & $39.73 \pm 1.06$ & 1.060 & $0.957-1.175$ & 0.265 \\
\hline Prenatal BMI, Kg/m² & $25.97 \pm 3.11$ & $26.07 \pm 3.08$ & 1.010 & $0.975-1.046$ & 0.593 \\
\hline \multicolumn{6}{|l|}{ Parity } \\
\hline Primiparous & $703(76.7)$ & $407(82.2)$ & \multirow{2}{*}{0.714} & \multirow{2}{*}{$0.541-0.941$} & \multirow{2}{*}{0.017} \\
\hline Multiparous & $213(23.3)$ & $88(17.8)$ & & & \\
\hline \multicolumn{6}{|l|}{ Delivery mode } \\
\hline Vaginal delivery & $596(65.1)$ & $303(61.2)$ & \multirow{2}{*}{1.117} & \multirow{2}{*}{$0.933-1.467$} & \multirow{2}{*}{0.173} \\
\hline Caesarean delivery & $320(34.9)$ & $192(38.8)$ & & & \\
\hline \multicolumn{6}{|l|}{ Maternal education } \\
\hline High school or below & $78(8.5)$ & $35(7.1)$ & \multirow{3}{*}{1.163} & \multirow{3}{*}{$0.936-1.444$} & \multirow{3}{*}{0.172} \\
\hline College graduate orbachelor degree & $675(73.7)$ & $360(72.7)$ & & & \\
\hline Graduate or above & $163(17.8)$ & $100(20.2)$ & & & \\
\hline Work full-time during the pregnancy & & & & & \\
\hline Yes & $802(87.6)$ & $450(90.9)$ & 704 & O 1800 & 0050 \\
\hline No & $114(12.4)$ & $45(9.1)$ & 0.704 & $0.489-1.012$ & 0.058 \\
\hline Household income, RMB/year & & & & & \\
\hline$<¥ 50,000$ & $52(5.7)$ & $32(6.5)$ & & & \\
\hline$¥ 50,000-100,000$ & $278(30.3)$ & $153(30.9)$ & 0947 & $0829-1082$ & 0423 \\
\hline$¥ 100,000-200,000$ & $411(44.9)$ & $223(45.0)$ & 0.947 & $0.829-1.082$ & 0.423 \\
\hline$>¥ 200,000$ & $175(19.1)$ & $87(17.6)$ & & & \\
\hline Living with parents & & & & & \\
\hline Yes & $697(76.1)$ & $361(72.9)$ & & & \\
\hline No & $361(23.9)$ & $134(26.1)$ & 1.181 & $0.920-1.516$ & 0.191 \\
\hline Drinking or smoking during pregnanc & & & & & \\
\hline Yes & $9(0.98)$ & $6(1.21)$ & 0809 & $0286-2285$ & 0689 \\
\hline No & $907(99.02)$ & 489 (98.79) & 0.809 & $0.286-2.285$ & 0.689 \\
\hline Maternity leave time & & & & & \\
\hline$<4$ months & $207(22.6)$ & $115(23.2)$ & & & \\
\hline $4 \sim 6$ months & $558(60.9)$ & $310(62.6)$ & 0902 & $0807-1049$ & (1) \\
\hline$>6$ months & $41(4.5)$ & $25(5.1)$ & 0.902 & $0.80 /-1.049$ & 0.211 \\
\hline Without work & $110(12.0)$ & $45(9.1)$ & & & \\
\hline Intended duration of breastfeeding & & & & & \\
\hline$<6$ months & $85(9.3)$ & $78(15.8)$ & & & \\
\hline $6 \sim 12$ months & $376(41.0)$ & $194(39.2)$ & 0.927 & $0.867-0991$ & 0.026 \\
\hline$>12$ months & $455(49.7)$ & $223(45.0)$ & & & \\
\hline Prenatal education attendance & & & & & \\
\hline None & $182(19.9)$ & $124(25.1)$ & & & \\
\hline Few & $372(40.6)$ & $193(39.0)$ & 0001 & 0704 & 0019 \\
\hline Almost all & $231(25.2)$ & $116(23.4)$ & 0.891 & $0.794-1.000$ & 0.049 \\
\hline All & $131(14.3)$ & $62(12.5)$ & & & \\
\hline Neonatal birth weight, $\mathrm{g}$ & $3449.80 \pm 379.82$ & $3442.76 \pm 373.27$ & 1.000 & $1.000-1.000$ & 0.738 \\
\hline CV-BSES scores & $118.54 \pm 19.44$ & $100.27 \pm 24.08$ & 0.962 & $0.956-0.968$ & 0.000 \\
\hline Early skin-to-skin contact and suckling & & & & & \\
\hline Within an hour after birth & $805(87.8)$ & $402(81.2)$ & 1678 & 12420266 & م001 \\
\hline An hour after birth & $111(12.2)$ & $93(18.8)$ & 1.678 & $1.242-2.266$ & 0.001 \\
\hline Using a pacifier & & & & & \\
\hline Yes & $24(2.6)$ & $19(3.8)$ & 0674 & $0365-1243$ & 0207 \\
\hline No & $892(97.4)$ & $476(96.2)$ & 0.674 & $0.365-1.243$ & 0.207 \\
\hline Perception of breast milk supply & & & & & \\
\hline Oversupply & $111(12.1)$ & $107(21.6)$ & & & \\
\hline Normal & $723(78.9)$ & $357(72.1)$ & 0.584 & $0.462-0.737$ & 0.000 \\
\hline Low supply & $82(9.0)$ & $31(6.3)$ & & & \\
\hline Early formula supplementation & & & & & \\
\hline Yes & $278(30.3)$ & $248(50.1)$ & 2304 & $1830-2887$ & 00000 \\
\hline No & $638(69.7)$ & $247(49.9)$ & 2.304 & $1.839-2.887$ & 0.000 \\
\hline Continued & & & & & \\
\hline
\end{tabular}




\begin{tabular}{|c|c|c|c|c|c|}
\hline \multirow[b]{2}{*}{ Variable } & \multicolumn{2}{|c|}{ Exclusive breastfeeding } & \multirow[b]{2}{*}{ OR } & \multirow[b]{2}{*}{$95 \%$ CI } & \multirow[b]{2}{*}{ P-value } \\
\hline & Yes, $\mathrm{N}=916,(\%)$ & No, $N=495,(\%)$ & & & \\
\hline \multicolumn{6}{|c|}{ Abnormal nipples } \\
\hline Yes & $57(6.2)$ & $98(19.8)$ & \multirow{2}{*}{3.720} & \multirow{2}{*}{$2.628-5.266$} & \multirow{2}{*}{0.000} \\
\hline No & $859(93.8)$ & $397(80.2)$ & & & \\
\hline \multicolumn{6}{|c|}{ Cracked or sore nipples } \\
\hline Yes & $123(13.4)$ & $93(18.8)$ & \multirow{2}{*}{1.492} & \multirow{2}{*}{$1.111-2.003$} & \multirow{2}{*}{0.008} \\
\hline No & $793(86.6)$ & $402(81.2)$ & & & \\
\hline \multicolumn{6}{|l|}{ Mastitis } \\
\hline Yes & $140(15.3)$ & $123(24.8)$ & \multirow{2}{*}{0.546} & \multirow{2}{*}{$0.416-0.716$} & \multirow{2}{*}{0.000} \\
\hline No & $776(84.7)$ & $372(65.2)$ & & & \\
\hline \multicolumn{6}{|c|}{ Neonatal jaundice } \\
\hline Yes & $183(20.0)$ & $151(30.5)$ & \multirow{2}{*}{0.569} & \multirow{2}{*}{$0.443-0.731$} & \multirow{2}{*}{0.000} \\
\hline No & $733(80.0)$ & $344(69.5)$ & & & \\
\hline
\end{tabular}

Table 1. Socio-demographic, clinical and breastfeeding characteristics of the participants.

Decision tree analysis. The decision tree resulting from Exhaustive Chi-squared Automatic Interaction Detection (exhaustive CHAID) analysis is shown in Fig. 2. This decision tree has a depth of four levels from the root node and 18 nodes, including 11 terminal nodes. Each node represents the probability of exclusive breastfeeding at two months postpartum for the corresponding branches. Based on the results of univariate analysis, ten significant predictors were found and were used to construct the decision tree model. Six significant predictors were successively selected to define further branches: the Chinese version of Breastfeeding Self-Efficacy Scale (CV-BSES) score, abnormal nipples, early formula supplementation, mastitis, neonatal jaundice, cracked or sore nipples and intended duration of breastfeeding.

The root variable in the decision tree is exclusive breastfeeding at two months postpartum. The variable that produces the first split in the decision tree is the CV-BSES score $\left(\chi^{2}=216.981 ; \mathrm{p}<0.001 ; \mathrm{df}=3\right)$. At the second division, four branches appear in the decision tree: the first was abnormal nipples, followed by early formula supplementation, mastitis and neonatal jaundice. At the third division, cracked or sore nipples, intended duration of breastfeeding and early formula supplementation were important predictors associated with the risk of discontinuing exclusive breastfeeding prematurely. The tree shows that participants at a higher risk of discontinuing exclusive breastfeeding prematurely are those who have a low CV-BSES score $(\leq 94)$ and abnormal nipples. The rate of EBF for these participants was only $15.4 \%$. Additionally, those with a CV-BSES score $>125$, without neonatal jaundice, and who did not supply formula prematurely were more likely to continue exclusive breastfeeding at least two months postpartum. They also had the highest rate of exclusive breastfeeding (90.4\%).

Comparison between the DT and LR Models. To examine the performance of the DT model in predicting early cessation of EBF before two months postpartum, we compared it to the conventional LR model. The LR model included risk indicators that all appeared in the DT model-that is, the CV-BSES score, abnormal nipples, early formula supplementation, mastitis and neonatal jaundice. On the other hand, variables overlooked by the LR model, such as cracked or sore nipples and intended duration of breastfeeding, were important predictors of early cessation of EBF for certain subgroups in the DT model. The comparison of the performance parameters of the DT and LR models is presented in Table 2. The decision tree had an estimation risk of 0.278 and a standard error of 0.12 , correctly classifying $73.1 \%$ of exclusive breastfeeding at two months postpartum. The sensitivity for this model was $75.5 \%$, and the specificity was $66.3 \%$. However, the accuracy, sensitivity and specificity of the LR model were $72.9 \%, 74.6 \%$ and $67.2 \%$, respectively. The DT model had a better classification accuracy and sensitivity than the LR model.

The area under the receiver operating characteristics (ROCs) curves (AUC) for the DT and LR models are shown in Fig. 3. The standard against which the sensitivity and specificity were calculated for both curves was EBF at two months postpartum. The areas under the curve were similar, $0.760(0.733,0.786)$ for the DT model and $0.763(0.737,0.789)$ for the LR model.

\section{Discussion}

This study showed that the DT model can successfully identify women at risk for early cessation of EBF before two months postpartum and showed similar or better performance than the LR model. The decision tree method has some advantages in model construction and application. First, the algorithm can divide consecutive data into the best predictive group, efficiently segmenting populations into meaningful subgroups ${ }^{20}$. Additionally, high-risk population subgroups can be identified. Interventions can be developed according to the specific needs of each subgroup, especially for vulnerable groups. In this study, the group with a CV-BSES score lower than 94 was the most important target population, in which the EBF rate was only $34 \%$. Additionally, the DT model described associations in the data by revealing important interactions among variables. Although abnormal nipples and cracked or sore nipples only had an effect on the group with a CV-BSES score lower than 94, they were meaningless in the higher CV-BSES score groups, indicating that the importance of each predictive variable varies in different subgroups. Furthermore, the DT model produced simple and visual tree graphics that can be easily applied in clinical practice. Therefore, DT can be easily used by clinical nurses and breastfeeding professionals. 


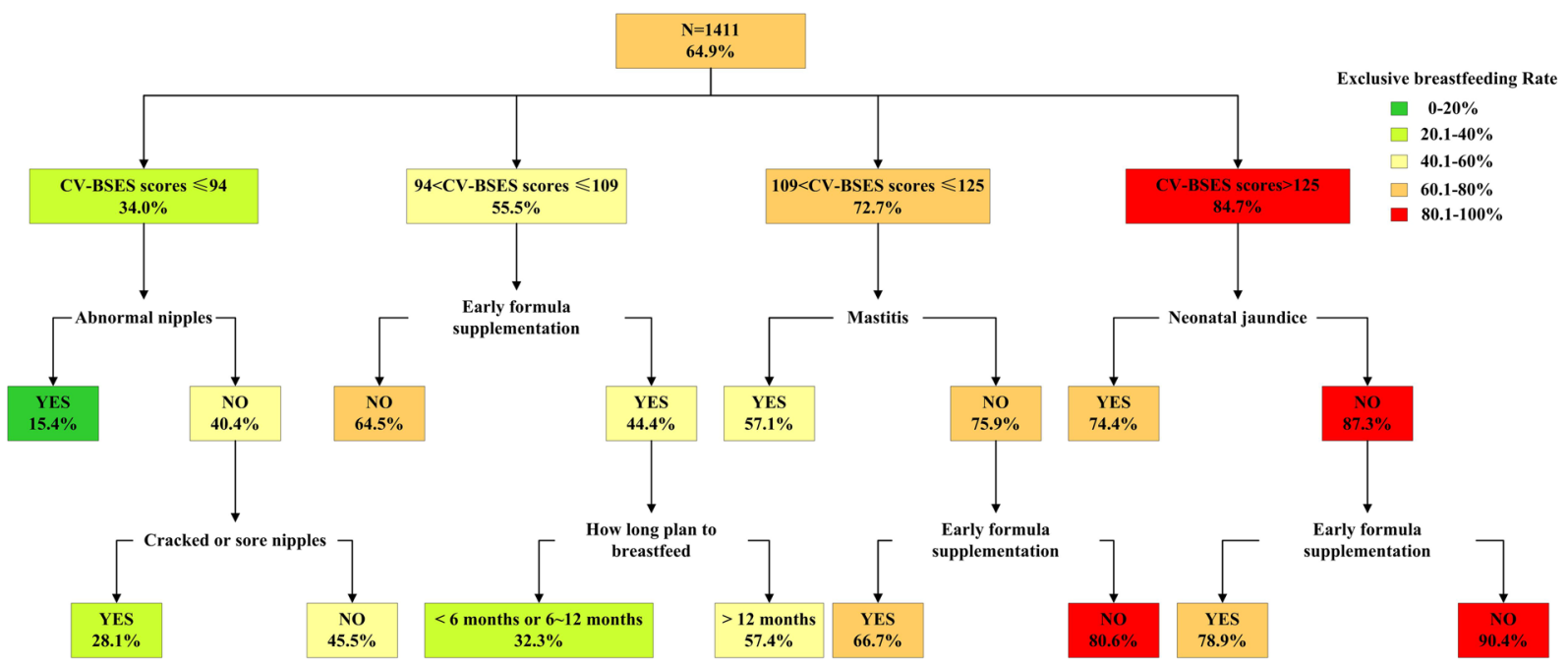

Figure 2. Decision tree predicting the risk for the early cessation of EBF at 2 months postpartum.

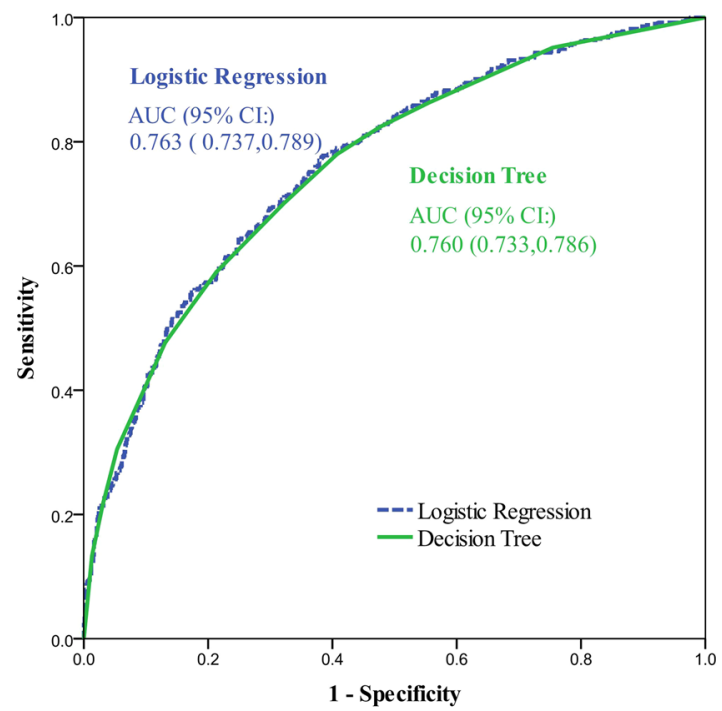

Figure 3. Receiver operating characteristic (ROC) curves for the decision tree and logistic regression models.

\begin{tabular}{|l|l|l|l|l|}
\hline \multirow{2}{*}{} & \multicolumn{3}{|l|}{ Decision Tree Model } & \multicolumn{2}{l|}{ Logistic Regression Model } \\
\cline { 2 - 6 } & $\begin{array}{l}\text { Predicted } \\
\text { positives }\end{array}$ & $\begin{array}{l}\text { Predicted } \\
\text { negatives }\end{array}$ & $\begin{array}{l}\text { Predicted } \\
\text { positives }\end{array}$ & $\begin{array}{l}\text { Predicted } \\
\text { negatives }\end{array}$ \\
\hline Diagnosed positives & $796(\mathrm{TP})$ & $120(\mathrm{FN})$ & $809(\mathrm{TP})$ & $107(\mathrm{FN})$ \\
\hline Diagnosed negatives & $259(\mathrm{FP})$ & $236(\mathrm{TN})$ & $276(\mathrm{FP})$ & $219(\mathrm{FN})$ \\
\hline Accuracy & 0.731 & 0.729 & \\
\hline Sensitivity & 0.755 & 0.746 & \\
\hline Specificity & 0.663 & 0.672 & \\
\hline
\end{tabular}

Table 2. Comparison of the performance parameters of the logistic regression and decision tree models. TP true positive, TN true negative, FP false positive, $\mathrm{FN}$ false negative a Accuracy $=(\mathrm{TP}+\mathrm{TN}) /$ $(\mathrm{TP}+\mathrm{FP}+\mathrm{TN}+\mathrm{FN}) \mathrm{b}$ Sensitivity $=\mathrm{TP} /(\mathrm{TP}+\mathrm{FN}) \mathrm{c}$ Specificity $=\mathrm{TN} /(\mathrm{TN}+\mathrm{FP})$.

The BSES score is one of the strongest predictors of early breastfeeding cessation. The maternal breast feeding self-efficacy is derived from the self-efficacy concept of Bandura ${ }^{26}$. According to Dennis, maternal breastfeeding self-efficacy refers to the mother's perceived ability to breastfeed her child that affects her decision on breastfeeding, such as whether to breastfeed, how much effort to place on breastfeeding and how to respond to challenges during breastfeeding. Mothers with a high self-efficacy are more inclined to breastfeed, persist when 
confronted with difficulties, are self-motivated and react positively to challenges ${ }^{27}$. Thus, the BSES score can affect breastfeeding initiation, duration, and exclusivity, with a higher score indicating a higher rate of EBF and better performance in breastfeeding practices. This finding is consistent with findings from other cohort studies ${ }^{28,29}$. With each 10-point increase in the BSES score, the exclusive breastfeeding rate increased by approximately 12 to 20 percent in this study. Mothers who had a low score were more likely to give up exclusive breastfeeding prematurely when they faced difficulties, such as abnormal, cracked or sore nipples. Many studies have applied the BSES score to prenatal measurements and have suggested that it can significantly predict exclusive breastfeeding postpartum $^{30,31}$. Thus, clinical nurses and breastfeeding professionals can use the BSES as a practical tool for prenatal and postnatal breastfeeding assessments, while the short form of BSES is more suitable and acceptable. Further research is warranted to provide more insight on this topic.

Based on the DT analysis, early formula supplementation is a widespread risk factor in many subgroups. In this study, $37.3 \%$ of neonates were supplied formula during hospitalization, a finding that is consistent with findings in previous breastfeeding studies in China ${ }^{32,33}$. The cause of this phenomenon is tightly related to the development status in China. With China's accelerating urbanization, economic development, fast pace of life and other changes, breastfeeding practice has encountered many challenges. Particularly, the impact of the formula market should be seriously considered. Alarmingly, advertisements in the media have portrayed formula as being as good as, or better than, breast milk or as a lifestyle choice for the family. This misinformation can affect a mother's decision to breastfeed ${ }^{34}$. It is easy to choose formula supplementation when mothers and their families perceive "inadequate breastmilk production" in the first week. Additionally, the convenience of shopping (online and offline) is another important reason. Presently, there are many ways to purchase formula, such as overseas online shopping, overseas purchasing agents, and domestic shopping websites. Indeed, China is now the largest infant formula market in the world, with a projected annual growth rate of 20 percent. Even in 2009, when the global real gross domestic product was negative, the annual sales of infant formula rose $8 \%$ in constant value ${ }^{16}$. In this situation, baby-friendly hospitals should play a role in protecting, promoting and supporting breastfeeding. Clinical nurses and breastfeeding professionals should enhance breastfeeding education from the prenatal to postpartum periods and include the benefits, knowledge and skills of breastfeeding.

In summary, clinical nurses and breastfeeding professionals should improve their ability to identify women at risk for discontinuing EBF before two months postpartum and target their efforts to provide practical help in clinical practice.

In this study, a DT model of EBF with relatively high sensitivity and specificity was established that not only identified independent risk factors predictive of an early cessation of EBF but also shed light on the assessment and management of EBF. Further validation of these models in a different database is required to test the accuracy of their classifications. Thus, work is in progress to gather new information at multiple centres and with heterogeneous populations to build a new dataset to test our models to predict early cessation of EBF.

\section{Methods}

Ethics statement. All participants were informed that their data concerning socio-demographic characteristics, clinical characteristics and breastfeeding practices that were collected would only be used for this study. A statement to the participants of our commitment to protect their personal information was also provided. All participants signed a consent form that declared their voluntary agreement with all of the procedures involved in this study in accordance with the tenets of the Declaration of Helsinki. Ethics approval for the study was granted by the Medical Ethics Committee of Nanjing Maternity and Child Health Care Hospital (File No.2016-112).

Study population and study design. This population-based longitudinal study was conducted at a baby-friendly hospital with a delivery rate of 26,000 deliveries per year in Nanjing, China. Convenience sampling was used because of resource constraints from September 2016 to January 2017. Participants were recruited from the outpatient department of an antenatal clinic at 37 weeks of gestation. Participants were selected based on the following criteria: (1) were aged 18 years or older, (2) had a singleton pregnancy, (3) intended to breastfeed, (4) had no contraindications for breastfeeding, and (5) could communicate and complete the questionnaires. Some unqualified mothers were excluded due to the following: (1) they had a factor that could significantly interfere with breastfeeding, such as special treatment and medication, (2) they had a high-risk pregnancy (i.e., serious medical condition or known birth defect), or (3) the baby was not discharged home with the mother. Due to the large sample size recommended by data mining technology, this study planned to enrol 1,500 participants.

Data collection at three stages. Potential predictors for breastfeeding were identified from published guidelines, systematic reviews and primary studies, including the three categories of socio-demographic characteristics, clinical characteristics and breastfeeding practices. According to the follow-up time, the predictors were measured by self-made questionnaires.

Time 1: 37 weeks of gestation. At 37 weeks of gestation at the antenatal clinic, participants were interviewed at a convenient location. After providing informed consent, eligible participants were asked by the research assistant to complete a socio-demographic questionnaire including their age, BMI, parity, education, employment status, maternity leave time, smoking and drinking behaviour, household income, and living and housing situation.

Time 2: follow-up within 3-5 days postpartum. All participants were interviewed in the postnatal ward after giving birth by a breastfeeding consultant at 3-5 days postpartum. Participants were asked to complete two-part questionnaires. Part A collected (1) clinical characteristics: delivery mode, perinatal complications, maternal medication use, and neonatal weight; (2) breastfeeding practices: prenatal education attendance, intended duration of breastfeeding, early skin-to-skin contact and suckling, pacifier use, early formula supplementation, 
abnormal nipples (flat, inverted or very large nipples), cracked or sore nipple, perception of breast milk supply, mastitis and neonatal jaundice. Part B was the Chinese version of Breastfeeding Self-Efficacy Scale (CV-BSES) ${ }^{35}$, which measured maternal breastfeeding attitudes and knowledge.

The CV-BSES is a 30 -item self-report instrument that has been psychometrically tested using a population-based sample of 186 breast-feeding mothers in China. All of the items are preceded by the phrase 'I am able to always ...' and are answered on a 5-point Likert scale ranging from 1 (not at all confident) to 5 (very confident). These ratings are summed into a total score ranging from 30 to 150, with a higher total score indicating a higher level of maternal breast-feeding self-efficacy. The Cronbach's $\alpha$ coefficient of this instrument was 0.93 , suggesting good internal consistency ${ }^{35}$.

Time 3: follow-up at two months postpartum. At two months postpartum, each woman was contacted by telephone to collect breastfeeding information by the research assistant. The main aim of this interview was to determine the mothers' breastfeeding status. At the same time, the occurrence of cracked or sore nipples, mastitis and neonatal jaundice at two months postpartum were recorded. The time point of two months was chosen because the CV-BSES score was an important predictor in this study and proved to show good predictive performance for breastfeeding practice between 4 and 8 weeks postpartum ${ }^{35}$.

All of the research assistants and breastfeeding consultants who participated in the study were trained together and were given detailed instructions and explanations about the completion of questionnaires and outcome measures. A standardized interview schedule was used to maintain consistency.

Statistical analysis. All data were analysed using the IBM SPSS 20.0 software package. Continuous data were compared using t-tests, and discrete count data were compared using $\chi^{2}$ test. Based on the results of univariate analysis, statistically significant variables were applied to construct a decision tree model and logistic regression model to assess the factors associated with breastfeeding cessation risk. In the DT model, the algorithm used was Exhaustive Chi-squared Automatic Interaction Detection or exhaustive CHAID. Chi squared test was used based on the minimum $P$ value. Branching stopped when there were no risk indicators with a p-value less than 0.1 for division. The minimum sample size for each leaf (node) was specified as $n=50$. The binary logistic regression method was used for multivariate analysis and establishment of the risk assessment model. An 'entry' approach was used to construct the LR model. A p value $<0.05$ was considered statistically significant. The performance of the predictive models was determined using AUC, accuracy, sensitivity and specificity. In both models, the predicted probabilities were the output and were used to create the curves. The higher was the AUC, the better performance the model predicted. The maximum AUC value is 1.0, indicating a perfect test. By contrast, an AUC value of 0.5 indicates no difference.

Received: 8 January 2019; Accepted: 12 February 2020;

Published online: 21 April 2020

\section{References}

1. in Guideline: Protecting, Promoting and Supporting Breastfeeding in Facilities Providing Maternity and Newborn Services (World Health Organization Copyright (c) World Health Organization 2017., 2017).

2. Breastfeeding and the Use of Human Milk. Pediatrics 129, e827-e841, https://doi.org/10.1542/peds.2011-3552 (2012).

3. Smithers, L. G., Kramer, M. S. \& Lynch, J. W. Effects of Breastfeeding on Obesity and Intelligence: Causal Insights From Different Study Designs. JAMA pediatrics 169, 707-708, https://doi.org/10.1001/jamapediatrics.2015.0175 (2015).

4. Victora, C. G. et al. Breastfeeding in the 21st century: epidemiology, mechanisms, and lifelong effect. The Lancet 387, 475-490, https://doi.org/10.1016/s0140-6736(15)01024-7 (2016).

5. McGuire, S., Centers for Disease Control and Prevention. 2013. Strategies to Prevent Obesity and Other Chronic Diseases: The CDC Guide to Strategies to Support Breastfeeding Mothers and Babies. Atlanta, GA: U.S. Department of Health and Human Services, 2013. Advances in nutrition 5, 291-292, https://doi.org/10.3945/an.114.005900 (2014).

6. Kramer, M. S. \& Kakuma, R. Optimal duration of exclusive breastfeeding. The Cochrane database of systematic reviews, CD003517, https://doi.org/10.1002/14651858.CD003517.pub2 (2012).

7. Colombo, L. et al. Breastfeeding Determinants in Healthy Term Newborns. Nutrients 10, https://doi.org/10.3390/nu10010048 (2018).

8. Dashti, M., Scott, J. A., Edwards, C. A. \& Al-Sughayer, M. Predictors of breastfeeding duration among women in Kuwait: results of a prospective cohort study. Nutrients 6, 711-728, https://doi.org/10.3390/nu6020711 (2014).

9. Waits, A., Guo, C. Y. \& Chien, L. Y. Evaluation of factors contributing to the decline in exclusive breastfeeding at 6 months postpartum: The 2011-2016 National Surveys in Taiwan. Birth 45, 184-192, https://doi.org/10.1111/birt.12340 (2018).

10. Nasser, A. et al. Predictors of Continued Breastfeeding at One Year among Women Attending Primary Healthcare Centers in Qatar: A Cross-Sectional Study. Nutrients 10, https://doi.org/10.3390/nu10080983 (2018).

11. Hawkins, S. S., Griffiths, L. J., Dezateux, C. \& Law, C. The impact of maternal employment on breast-feeding duration in the UK Millennium Cohort Study. Public health nutrition 10, 891-896, https://doi.org/10.1017/s1368980007226096 (2007).

12. Higgins, T. M. et al. Effects of cigarette smoking cessation on breastfeeding duration. Nicotine \& tobacco research: official journal of the Society for Research on Nicotine and Tobacco 12, 483-488, https://doi.org/10.1093/ntr/ntq031 (2010).

13. Lumbiganon, P. et al. Antenatal breastfeeding education for increasing breastfeeding duration. The Cochrane database of systematic reviews 12, Cd006425, https://doi.org/10.1002/14651858.CD006425.pub4 (2016).

14. Mok, E. et al. Decreased full breastfeeding, altered practices, perceptions, and infant weight change of prepregnant obese women: a need for extra support. Pediatrics 121, e1319-1324, https://doi.org/10.1542/peds.2007-2747 (2008).

15. Jaafar, S. H., Ho, J. J., Jahanfar, S. \& Angolkar, M. Effect of restricted pacifier use in breastfeeding term infants for increasing duration of breastfeeding. The Cochrane database of systematic reviews, Cd007202, https://doi.org/10.1002/14651858.CD007202.pub4 (2016).

16. Rollins, N. C. et al. Why invest, and what it will take to improve breastfeeding practices? The Lancet 387, 491-504, https://doi. org/10.1016/s0140-6736(15)01044-2 (2016).

17. Yang, Z. et al. Breastfeeding rates in China: a cross-sectional survey and estimate of benefits of improvement. The Lancet 388, S47, https://doi.org/10.1016/s0140-6736(16)31974-2 (2016).

18. Liu, J. et al. Social and demographic determinants for breastfeeding in a rural, suburban and city area of South East China. Contemporary nurse 45, 234-243, https://doi.org/10.5172/conu.2013.45.2.234 (2013). 
19. Batterham, P. J., Christensen, H. \& Mackinnon, A. J. Modifiable risk factors predicting major depressive disorder at four year followup: a decision tree approach. BMC psychiatry 9,75 , https://doi.org/10.1186/1471-244X-9-75 (2009).

20. Kobayashi, D., Takahashi, O., Arioka, H., Koga, S. \& Fukui, T. A prediction rule for the development of delirium among patients in medical wards: Chi-Square Automatic Interaction Detector (CHAID) decision tree analysis model. The American journal of geriatric psychiatry: official journal of the American Association for Geriatric Psychiatry 21, 957-962, https://doi.org/10.1016/j. jagp.2012.08.009 (2013).

21. Ramos-Medina, R. et al. New decision-tree model for defining the risk of reproductive failure. American journal of reproductive immunology 70, 59-68, https://doi.org/10.1111/aji.12098 (2013).

22. Piper, M. E., Loh, W. Y., Smith, S. S., Japuntich, S. J. \& Baker, T. B. Using decision tree analysis to identify risk factors for relapse to smoking. Substance use \& misuse 46, 492-510, https://doi.org/10.3109/10826081003682222 (2011).

23. Rafiq, M. et al. Falls in the elderly were predicted opportunistically using a decision tree and systematically using a database-driven screening tool. Journal of clinical epidemiology 67, 877-886, https://doi.org/10.1016/j.jclinepi.2014.03.008 (2014).

24. Song, Y. Y. \& Lu, Y. Decision tree methods: applications for classification and prediction. Shanghai archives of psychiatry 27, 130-135, https://doi.org/10.11919/j.issn.1002-0829.215044 (2015).

25. Fernandez, L., Mediano, P., Garcia, R., Rodriguez, J. M. \& Marin, M. Risk Factors Predicting Infectious Lactational Mastitis: Decision Tree Approach versus Logistic Regression Analysis. Maternal and child health journal 20, 1895-1903, https://doi. org/10.1007/s10995-016-2000-6 (2016).

26. Bandura, A. Self-efficacy: toward a unifying theory of behavioral change. Psychological review 84, 191-215 (1977).

27. Dennis, C. L. Theoretical underpinnings of breastfeeding confidence: a self-efficacy framework. Journal of human lactation: official journal of International Lactation Consultant Association 15, 195-201, https://doi.org/10.1177/089033449901500303 (1999).

28. Wu, D. S., Hu, J., McCoy, T. P. \& Efird, J. T. The effects of a breastfeeding self-efficacy intervention on short-term breastfeeding outcomes among primiparous mothers in Wuhan, China. Journal of Advanced Nursing 70, 1867-1879, https://doi.org/10.1111/ jan.12349 (2014).

29. Chan, M. Y., Ip, W. Y. \& Choi, K. C. The effect of a self-efficacy-based educational programme on maternal breast feeding selfefficacy, breast feeding duration and exclusive breast feeding rates: A longitudinal study. Midwifery 36, 92-98, https://doi. org/10.1016/j.midw.2016.03.003 (2016).

30. Brandao, S. et al. The breastfeeding self-efficacy scale-short form: Psychometric characteristics in Portuguese pregnant women. Midwifery 66, 49-55, https://doi.org/10.1016/j.midw.2018.07.014 (2018).

31. Noel-Weiss, J., Rupp, A., Cragg, B., Bassett, V. \& Woodend, A. K. Randomized controlled trial to determine effects of prenatal breastfeeding workshop on maternal breastfeeding self-efficacy and breastfeeding duration. Journal of obstetric, gynecologic, and neonatal nursing: JOGNN 35, 616-624, https://doi.org/10.1111/j.1552-6909.2006.00077.x (2006).

32. Tang, L. et al. Widespread usage of infant formula in China: a major public health problem. Birth 41, 339-343, https://doi. org/10.1111/birt.12132 (2014).

33. Tang, L., Binns, C. W. \& Lee, A. H. Infant formula crisis in china: a cohort study in sichuan province. Journal of health, population, and nutrition 33, 117-122 (2015).

34. Chen, Y. C., Chang, J. S. \& Gong, Y. T. A Content Analysis of Infant and Toddler Food Advertisements in Taiwanese Popular Pregnancy and Early Parenting Magazines. Journal of human lactation: official journal of International Lactation Consultant Association 31, 458-466, https://doi.org/10.1177/0890334415576513 (2015).

35. Dai, X. \& Dennis, C. L. Translation and validation of the Breastfeeding Self-Efficacy Scale into Chinese. Journal of midwifery \& women's health $48,350-356$ (2003).

\section{Acknowledgements}

This work was supported, in part, by the Medical Science and Technology Development Foundation of Nanjing Department of Health (YKK15152). We thank Dr. Zhen Li at Nanjing Agricultural University for useful advice.

Author contributions

C.S. and Y.T. designed the study. L.D., Y.Z. and Y.W. collected the data. Y.W. and J.W. conducted the statistical analysis. Y.W. wrote the report. C.S. and Y.T. revised the report. All of the authors reviewed the report and approved the final version.

\section{Competing interests}

The authors declare no competing interests.

\section{Additional information}

Correspondence and requests for materials should be addressed to Y.T.

Reprints and permissions information is available at www.nature.com/reprints.

Publisher's note Springer Nature remains neutral with regard to jurisdictional claims in published maps and institutional affiliations.

(c) (i) Open Access This article is licensed under a Creative Commons Attribution 4.0 International

License, which permits use, sharing, adaptation, distribution and reproduction in any medium or format, as long as you give appropriate credit to the original author(s) and the source, provide a link to the Creative Commons license, and indicate if changes were made. The images or other third party material in this article are included in the article's Creative Commons license, unless indicated otherwise in a credit line to the material. If material is not included in the article's Creative Commons license and your intended use is not permitted by statutory regulation or exceeds the permitted use, you will need to obtain permission directly from the copyright holder. To view a copy of this license, visit http://creativecommons.org/licenses/by/4.0/.

(C) The Author(s) 2020 